\section{A case of autoimmune limbic encephalitis in a patient with Behcet's disease}

\author{
Nehal Narayan, Andrew Whallett \\ Rheumatology Department, Russell's Hall \\ Hospital, West Midlands, UK
}

\begin{abstract}
Behcet's disease is well known to have neurological manifestations, most usually as a consequence of focal parenchymal lesions or vascular thrombosis. We report a case of autoimmune limbic encephalitis in a patient with Behcet's disease, that was highly responsive to immunoglobulins and steroid, which ultimately prevented mortality, and reduced comorbidity. We also review the investigation and management of non-paraneoplastic, or autoimmune limbic encephalitis.
\end{abstract}

\section{Introduction}

Behcet's disease is a multisystem disorder characterized by recurrent orogenital ulceration and occlusive vasculitis. ${ }^{1}$ Neurological manifestations have been reported to occur in as many as $20 \%$ of patients with Behcet's disease, with focal parenchymal lesions and complications of vascular thrombosis being the most common abnormalities. ${ }^{2}$ In the literature to date, there is only one previous reported case of limbic encephalitis in a patient with Behcet's/relapsing polychondritis overlap. ${ }^{3}$

Here, we describe a patient with known Behcet's disease who developed limbic encephalitis. All other known causes of limbic encephalitis had been excluded, suggesting a possible association between the autoimmune process of limbic encephalitis and Behcet's. We also briefly review the differential diagnosis of limbic encephalitis and the management of non-paraneoplastic or autoimmune limbic encephalitis.

\section{Case Report}

Our patient is a 50 year old Caucasian woman, with a history of Behcet's for over 10 years, manifesting as recurrent oral and genital ulceration, with uveitis, well controlled on ciclosporin $125 \mathrm{mg}$ once daily. At her last review 3 months previously, she had been fit and well, free from ulcers, with no evidence of ocular inflammation, although mood was slightly low. She presented to the emergency department with a 2 week history of acute confusion and increasing drowsiness. On admission, Glasgow Coma Scale (GCS) score was $13 / 15$, with inappropriate speech and eye opening to voice only. Rest of examination was unremarkable, except for minimal oral ulceration. Bloods demonstrated elevated C-reactive protein (CRP) at $48(<3 \mathrm{mg} / \mathrm{L})$, and new normocytic anemia. Computed tomography (CT) head was unremarkable. cerebrospinal fluid (CSF) microscopy was clear. CSF protein was marginally elevated at $0.48 \quad(>0.4 \mathrm{~g} / \mathrm{L})$. Ciclosporin was stopped, and she was commenced on treatment for infective meningoencephalitis (which included high dose intravenous antiviral treatment in the form of aciclovir), without improvement-indeed by day 4 of antimicrobials, GCS score had dropped to 11/15 and she had developed a left hemiparesis. Cerebrospinal fluid viral polymerase chain reaction (PCR) came back negative. Blood viral serology entirely negative also, with evidence of pre-existing immunity to EBV and CMV. A MRI demonstrated confluent hyperintensity in the temporal lobes, consistent with limbic encephalitis (Figure 1), and the patient was commenced on intravenous hydrocortisone, and received a course of intravenous immunoglobulin. By day 3 of hydrocortisone, GCS score had come back to 14/15, with the patient having confused speech. Her left hemiparesis began to improve. Given the association of limbic encephalitis with malignancy, full body CT and positron emission tomography-CT (PET-CT) were performed. These did not demonstrate any evidence of malignancy. Likewise, tests for paraneoplastic auto-antibodies were negative. There is increasing evidence to suggest some forms of limbic encephalitis have a non-paraneoplastic autoimmune cause..$^{4-6}$ Tests for the most commonly reported non-paraneoplastic antibodies associated with limbic encephalitis were also negative (Table 1).

An EEG confirmed epileptiform activity. She now continues on reducing course of prednisolone, and azathioprine has been initiated. CRP is now less than 3 . She continues to make a neurological recovery.

\section{Discussion}

Limbic encephalitis refers to encephalitis, with the characteristic finding of inflammation in the temporal lobes. Presentation includes confusion, memory loss, focal neurology and seizures. The mainstay of management is treatment of the underlying cause. ${ }^{7}$

Historically, the most commonly reported cause of limbic encephalitis is viral infection. The most commonly recognized infective
Correspondence: Nehal Narayan, The Botnar Research Center, Windmill Road, Headington, 0xford, OX3 7LD, UK.

Tel.: +44.079.443.55406.

E-mail: nehalpsn@doctors.org.uk

Key words: Encephalitis; neurologic manifestations; Behcet's disease.

Contributions: NN, responsible for the writing of this article, and involved in the diagnosis and management of this patient's case, playing a major role in the care of this patient; AW, responsible for amending and reviewing any content submitted for publication, and responsible for leading the management of this patient in this case as her named consultant.

Conflict of interest: the authors declare no potential conflict of interest.

Received for publication: 10 December 2014. Revision received: 22 March 2015.

Accepted for publication: 27 March 2015.

This work is licensed under a Creative Commons Attribution NonCommercial 3.0 License (CC BYNC 3.0).

(OCopyright N. Narayan and A. Whallett, 2015 Licensee PAGEPress, Italy

Rheumatology Reports 2015; 7:5751

doi:10.4081/rr.2015.5751

agent is herpes simplex. Treatment is with high dose antivirals. The other commonly reported form of limbic encephalitis is as part of a paraneoplastic phenomenon, alleviated by treatment of the underlying neoplasia. However, over recent years, there have been reports of a number of cases of limbic encephalitis occurring in the absence of infection or malignancy. These are termed nonparaneoplastic limbic encephalitis or simply autoimmune limbic encephalitis, responding to prompt immunosuppression. ${ }^{7}$ In these cases, it has been proposed that the encephalitis arises from an autoimmune process with the involvement of presently unidentified target antigens. Recently, case series have demonstrated the association of the non-paraneoplastic anti-neuronal antibodies anti-voltage gated potassium channel and anti-glutamic acid decarboxylase with limbic encephalitis. ${ }^{4,5}$ Our patient, with no evidence of malignancy or infection, would therefore fit into the category of non-paraneoplastic limbic encephalitis, possibly with involvement of a currently unidentified auto antigen. This may point to a link between the autoimmune process of Behcet's and limbic encephalitis, a previously unreported association.

In all reported cases of non-paraneoplastic 


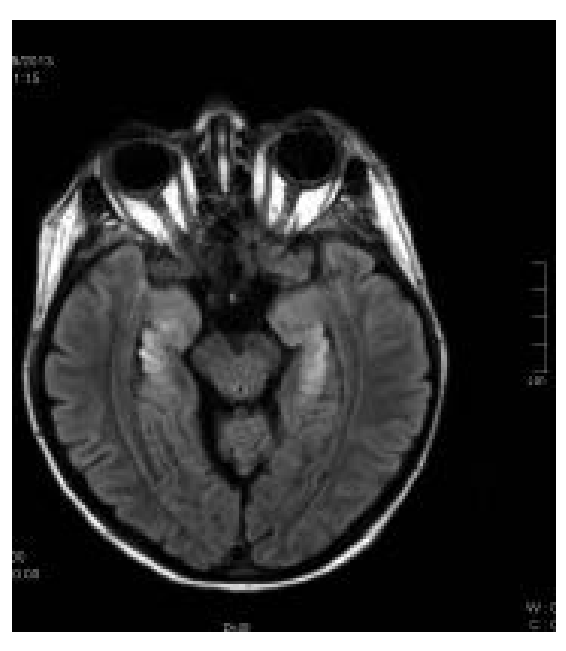

Figure 1. Magnetic resonance imaging FLAIR sequence of our patient demonstrating symmetrical enhancement in the hippocampal regions bilaterally (located in medial portion of temporal lobes) characteristic of limbic encephalitis.

limbic encephalitis, the management has been consistent. ${ }^{7}$ All patients with suspected nonparaneoplastic limbic encephalitis have a lumbar puncture, to rule out infection, and thorough infection screen, including viral PCR of CSF. In addition, an MRI scan is needed to confirm the characteristic changes of hyperintensity in the limbic regions. Further, thorough screening for paraneoplastic autoantibodies is required (as demonstrated by our case). Due to the association of limbic encephalitis with early cancer, there is increasing evidence to support the use of PET-CT if other body imaging is negative, to detect any malignancy. ${ }^{7}$ Treatment involves high dose steroid (methylprednisolone, or in our case hydrocortisone) and intravenous immunoglobulins. ${ }^{7}$ Further study into both autoimmunity in Behcet's and non-paraneoplastic limbic encephalitis is required to identify pertinent auto-antigens, which may also give further clues as to disease pathogenesis in Behcet's. The use of

Table 1. Autoantibody testing in our patient to look for evidence of paraneoplastic syndrome or known non-paraneoplastic limbic encephalitis associated auto-antibodies.

Result

Paraneoplastic auto-antibodies (and most commonly associated cancer)

Anti-Hu (small cell lung cancer)

Anti-Ri (breast or small cell lung cancer)

(most commonly testicular cancer, but also non small cell lung cancer)

Anti-CV2 (non small cell lung cancer)

Anti-Yo (gynaecological tumors)

Anti-NMDA (N-methyl D-aspartate) Receptor (ovarian teratoma)

Non-paraneoplastic auto-antibodies associated with limbic encephalitis

Anti-Voltage Gated Potassium Channel

Anti-Glutamic Acid Decarboxylase

Negative

Negative

Negative

Negative

ciclosporin in patients with Behcet's has also been postulated to be associated with an increased incidence of central nervous system manifestations of Behcet's, ${ }^{8}$ although the mechanism for this remains to be elicited, and the association may reflect the inefficacy of ciclosporin to control neurological disease, rather than any neurotoxic effect.

\section{Conclusions}

Through recognizing the fact that autoimmune limbic encephalitis was on the differential of causes of encephalitis, we were able to prevent mortality and reduce morbidity in our patient's case. We hope this case therefore demonstrates the importance of recognizing this rare, but potentially devastating cause of limbic encephalitis. Furthermore, we suggest that there should be a raised index of suspicion of autoimmune encephalitis in those Behcet's patients presenting with features of encephalitis, regardless of presence of other features of Behcet's.

\section{References}

1. Sakane T, Takeno M, Suzuki N, Inabe G.
Behcet's disease. N Engl J Med 1999;341: 1284-91.

2. Al-Araji A, Kidd DP. Neuro-Behcet's disease: epidemiology, clinical characteristics and management. Lancet Neurol 2009;8: 192-204.

3. Kumar N, Leep Hunderfund AN, Kutzbach BR, et al. A limbic encephalitis MR imaging in a patient with Behcet's disease and relapsing polychondritis. Am J Neuroradiol 2009;30:e96.

4. Vincent A, Buckley CM, Schott JM, et al. Potassium channel antibody-associated encephalopathy: a potentially immunotherapy-responsive form of limbic encephalitis. Brain 2004;127:701-12.

5. Malter MP, Helmstaedter C, Urbach $\mathrm{H}$, et al. Antibodies to glutamic acid decarboxylase define a form of limbic encephalitis. Ann Neurol 2010;67:470-8.

6. Darnell RB, Posner JB. A new cause for limbic encephalopathy. Brain 2005; 128:1745-6.

7. Schott JM. Limbic encephalitis-a clinician's guide. Pract Neurol 2006;6:143-53.

8. Kötter I, Günaydin I, Batra M, et al. CNS involvement occurs more frequently in patients with Behçet's disease under cyclosporin A (CSA) than under other medications--results of a retrospective analysis of 117 cases. Clin Rheumatol 2006;25:482-6. 